\title{
Integrated Energy Multi-Objective Operation Optimization
}

\author{
Zhao Liu ${ }^{1}$, Mengjin $\mathrm{Hu}^{1}$, Xianlong Zhao ${ }^{1}$, Yongli Wang ${ }^{2}$, Suhang Yao $^{2}$, Yang Ma ${ }^{2 *}$, Minhan Zhou ${ }^{2}$, Fuhao Song \\ ${ }^{1}$ Economic and Technological Research Institute of State Grid Hebei Electric Power Co., Ltd, Hebei 050000, China \\ 2 Departments of Economics and Management, North China Electric Power University, Beijing 102200, China
}

\begin{abstract}
The Integrated Energy System (IES) offers a new approach to the energy dilemma. It introduces demand-side response into the Regional Integrated Energy System (RIES) and proposes an operational optimization model considering Integrated Demand Side Response (IDSR) in the framework of RIES. First of all, the typical cold thermallelectric RIES is used as a framework to establish the multi-energy flow model of RIES based on the energy flow relationships among energy production, conversion and consumption. Then, from the perspective of energy transfer, the potential to enhance renewable energy consumption by carrying out RIES to transfer the system energy flow is analyzed. Finally, the RIES operation optimization model considering IDSR is established with the goal of the economy of system operation, considering the system energy balance and the operating constraints of each energy device, and the solution algorithm is designed. Taking the RIES in a certain region as an example, the proposed method and model were verified computationally, and the results showed that IDR can effectively improve system economics and increase the level of renewable energy consumption.
\end{abstract}

\section{Introduction}

The rapid economic development makes human beings face the problems of fossil energy depletion and environmental pollution [1]. Regional integrated energy system (RIES) will unify and coordinate the operation and management of electrical energy, natural gas, thermal energy and various new energy sources, and the complementary use of various energy sources within the region can realize the gradual utilization of energy and improve energy utilization efficiency [2]. At present, China has already carried out the Tianjin Eco-city demonstration project of combined cooling/heating/ electricity supply and other multi-energy management projects, energy saving and emission reduction, and maximizing the use of renewable energy is the demand of establishing RIES. The rapid development of the Integrated Energy System (IES) has put forward a new solution for the elimination of renewable energy [3]. Regional Integrated Energy System (RIES), as a form of IES, is characterized by large load demand and various forms of energy use, and demand-side response in RIES can effectively improve the efficiency of energy use and enhance the level of renewable energy absorption, which is attracting more and more attention.

A large amount of literature has investigated the optimal operation of RIES, which can be divided into two categories: one is the simple single-objective optimization problem. In [4], a penalty factor for wind abandonment and net load fluctuation was included in the objective function to verify that the proposed model can effectively suppress net load fluctuation; in [5], a combined heat and power (CHP) unit with combined heat and power (CHP) as the objective function is a simple sum of the combined cost and environmental cost. The common single-objective planning has the flaw that it is difficult to coordinate multiple scheduling demands, and the introduction of penalty coefficients does not reflect the importance of a scheduling goal to the scheduling decision maker. The other type of problem is multi-objective optimization. This approach is highly subjective in the selection of weights and relies on the experience of the scheduling decision maker. In [6], a multi-objective weighted fuzzy planning method is used to solve the multi-objective scheduling problem in RIES, taking into account the operation cost of power-to-gas conversion; in [7], a hierarchical analysis entropy weight assignment method is used to complete the comprehensive evaluation of multiple indicators of power system. However, the complexity of the RIES structural model makes it difficult to solve the Pareto frontier accurately, and a lot of literature uses heuristic algorithms to solve this problem. In [8], the RIES multiobjective planning model is solved by combining the unordered genetic algorithm and the interior point method; in [9], the target approximation and quadratic sequence planning methods are used to effectively solve the multi-objective capacity optimization problem of distributed generation systems. However, the accuracy and efficiency of these algorithms are not high, and the selection of a reasonable optimization and solution filtering method is also required. In [10], demand-side management and energy storage systems are coordinated to improve the system's wind power consumption capacity and reduce pollution emissions. Literature [11] introduces demand-side management into the planning

\footnotetext{
*Corresponding author: m15210225501@163.com
} 
problem of electrical interconnected integrated energy systems, which can effectively flatten the multi-energy load profile. Therefore, it is necessary to consider the introduction of integrated demand response in RIES and study its impact on multiple objectives.

The above-mentioned literature has conducted relevant studies on the involvement of demand-side response in system operation in RIES. However, there is still a lack of research on the mechanism of improving system flexibility and economy by implementing IDR in RIES. The potential of IDR for energy transfer is still at the stage of qualitative analysis, and quantitative analysis is lacking. In this paper, based on the above problems, we first use a typical cold/heat/electric RIES as a framework to establish a multi-energy flow model of RIES based on the energy flow relationship among energy production, conversion and consumption. Then, from the perspective of energy transfer, we quantitatively analyzed the potential of transferring system energy and improving system flexibility by carrying out IDR. Finally, the RIES operation optimization model considering IDR is established and the solution algorithm is designed, taking the economy of system operation as the goal, the energy balance of the system and the operating constraints of each energy device into consideration. The calculation results show that IDR can effectively improve the system economy and flexibility and increase the level of renewable energy consumption.

\section{RIES multi-objective optimization model}

\subsection{RIES structure}

The structure of the RIES in the text is shown in Figure 1. The RIES can purchase energy from the external distribution network, the gas distribution network, which mainly contains wind turbines, power to gas (P2G), CHP, gas boilers and savings batteries. The energy demand on the customer side of the energy hub is for electricity, gas and heat, ignoring energy transmission processes and losses. In this paper, we consider three demand response methods, namely, load reduction, load shifting and load substitution. In this paper, we consider three demand response methods: load reduction, load shifting and alternative load. The load shifting method reduces the peak pressure by reducing the peak load; the load shifting method shifts a large amount of load to the windy period to consume wind power by changing the power consumption time sequence, and large factories can often provide a higher response capacity with a more obvious response effect; in the windy period, the electricity load can be used to replace the heat load to improve the wind power consumption.

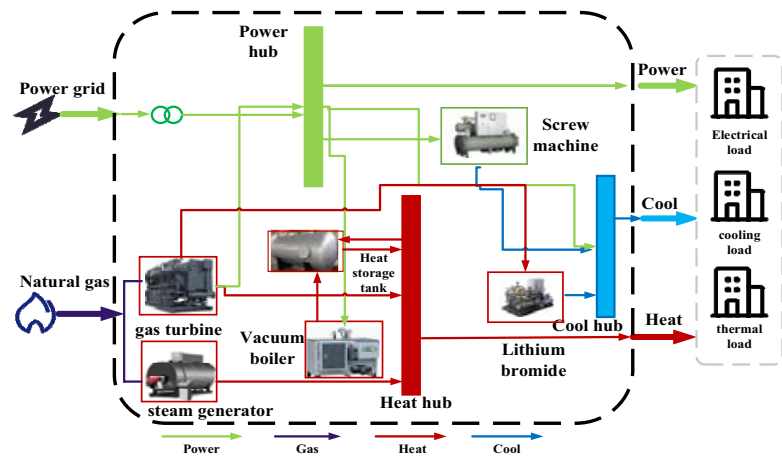

Fig.1 Structure map of a RIES

\subsection{RIES Multiple Energy Flow Model}

The energy conversion chain consists of Electric Heating (EH), Electric Cooling (EC), CCHP, Gas Boiler (GB) and Absorption Boiler (AB).

The energy consumption chain consists of electricity demand $E_{d}$, heat demand $H_{d}$ and cold demand $C_{d}$. Electricity demand can be supplied by renewable energy generation, external grid and CCHP; heat demand can be supplied by $\mathrm{EH}$ and $\mathrm{GB}$; and cold demand can be supplied by EC and AB. In RIES, the transformation and consumption processes belong to the demand side of energy.

The key to RIES multi-energy flow is to describe the energy conversion and distribution relationship of each energy conversion link. In this paper, two parameters, energy conversion coefficient $\eta$ and energy distribution coefficient $\alpha$, are used to describe the energy conversion and distribution relationships when building the RIES multi-energy flow model.

The conversion efficiency is the ratio of the output power $W_{o}$ to the input power $W_{i}$ which is recorded as the energy conversion coefficient $\eta$,

$$
\eta=W_{o} / W_{i}
$$

For example, for the electric heating link $(\mathrm{EH})$, the energy conversion factor $\eta_{H}^{E H}$ is the ratio of the output thermal power and the input electrical power.

For the energy distribution link, assume that its input power is $W_{i}$, output power is $W_{o 1}$ and $W_{02}$ respectively, and its energy distribution coefficients are $\alpha_{1}$ and $\alpha_{2}$, then there are:

$$
\begin{aligned}
& \alpha_{1}=W_{O 1} / W_{i} \\
& \alpha_{2}=W_{O 2} / W_{i}
\end{aligned}
$$

According to the input/output energy balance there are:

$$
\alpha_{1}+\alpha_{2}=1
$$

For example, for the power distribution link, part of the power output from the distribution system is directly distributed to the electric load, and part is distributed to the electric heating/cooling link, so the distribution coefficients are $\alpha_{E}^{E D}$ and $\alpha_{E}^{E U}$, and there are:

$$
\alpha_{E}^{E D}+\alpha_{E}^{E U}=1
$$


Assume that in the RIES system, the allocation factors for the RIES, EDS system to the electric load and the electric heating/cooling link are $\alpha_{E}^{E D}$ and $\alpha_{E}^{E U}$, the allocation factors for the electric heating and electric cooling links are $\alpha_{E}^{E H}$ and $\alpha_{E}^{E C}$, the allocation factors for the FDS system to the CCHP system and the gas boiler are $\alpha_{F}^{C C H P}$ and $\alpha_{F}^{G B}$, and the allocation factors for the CCHP direct heating and absorption chillers are $\alpha_{H}^{H D}$, $\alpha_{H}^{A B}$ respectively.

The energy supply of electricity and gas is expressed in terms of $V_{S}$ and is given as:

$V_{S}=\left[E_{S}, \mathrm{~F}_{S}\right]$

$V_{d}$ represents the cooling, heating and electrical energy requirements and is recorded as:

$$
V_{d}=\left[E_{d}, H_{d}, C_{d}\right]
$$

$\mathrm{T}$ denotes the energy transfer link, and according to conservation of energy, for the entire IES, there are:

$V_{d}=T V_{S}$

The energy flow relations obtained from energy transfer relations and conservation of energy are as follows.

For power transmission:

$$
E_{S} \alpha_{E}^{E D}+F_{S} \alpha_{F}^{C C H P} \eta_{E}^{C C H P}=E_{d}
$$

For heat transfer :

$$
\begin{aligned}
& E_{S} \alpha_{E}^{E U} \alpha_{E}^{E H} \eta_{H}^{C C H P}+F_{S} \alpha_{F}^{C C H P} \eta_{E}^{C C H P} \alpha_{E}^{E H} \eta_{H}^{E H} \\
& +F_{S} \alpha_{F}^{C C H P} \eta_{H}^{C C H P} \alpha_{H}^{H D}+F_{S} \alpha_{F}^{G B} \eta_{H}^{G B}=H_{d}
\end{aligned}
$$

For cold energy transmission :

$$
\begin{aligned}
& E_{S} \alpha_{E}^{E U} \alpha_{E}^{E C} \eta_{C}^{E C}+F_{S} \alpha_{F}^{C C H P} \eta_{E}^{C C H P} \alpha_{E}^{E C} \eta_{C}^{E C} \\
& +F_{S} \alpha_{F}^{C C H P} \eta_{H}^{C C H P} \alpha_{H}^{A B} \eta_{C}^{A B}=C_{d}
\end{aligned}
$$

For the distribution coefficients of the distribution links:

$$
\begin{aligned}
& \alpha_{E}^{E D}+\alpha_{E}^{E U}=1 \\
& \alpha_{E}^{E H}+\alpha_{E}^{E C}=1 \\
& \alpha_{F}^{C C H P}+\alpha_{F}^{G B}=1 \\
& \alpha_{H}^{H D}+\alpha_{H}^{A B}=1
\end{aligned}
$$

\section{Model Linearization and Multi- Objective Solving}

\subsection{Multi-Objective Solution Flow}

In this paper, according to the RIES model established above, we use a mature commercial software package (CPLEX) to solve the optimal solutions for three different objective functions, which constitute a set of multi-objective ideal solutions $\left(\mu_{C O S T}^{m i n}, F_{E N}^{m i n}, F_{W I N D}^{m i m}\right)$;

Then, the three objective functions are fuzzy with the help of the affiliation function, and the multi-objective optimization problem is scalarized by fuzzy weighting; next, a large number of Pareto non-inferior solutions are solved by refining the step size and traversing the weights; finally, the evidence inference decision method is used as the basis for filtering the optimal scheduling strategy to find a compromise solution.

\subsection{Multi-Objective Processing}

The fuzzy weighting method is used for multi-objective optimization. Firstly, the "descending half-trapezium" affiliation function of three optimization objectives is established, and the identification of three objective functions is omitted in the form of matrix description, which is:

$$
\mu(\mathrm{F})=\left\{\begin{array}{c}
1, F \leq F^{\mathrm{min}} \\
\frac{F^{\mathrm{min}}+\alpha F^{\mathrm{min}}-F}{\alpha F^{\min }}, \\
F^{\mathrm{min}}<F<F^{\min }+\alpha F^{\min } \\
0, F>F^{\min }>\alpha F^{\text {min }}
\end{array}\right.
$$

$\mu(F)$ is the satisfaction function of the objective function; $F^{m i n}$ is the multi-objective ideal point; $\alpha$ is the elastic satisfaction factor; and $\alpha F^{\text {min }}$ is the range of objective function growth acceptable to the scheduling decision maker.

The multi-objective optimization problem can then be transformed scalarly by summing the three satisfaction functions with the weight of a as a weight vector:

$$
\left\{\begin{array}{l}
\min a^{T} \mu(\mathrm{F})=\mathrm{a}_{1} \mu\left(\mathrm{F}_{C O S T}\right)+\mathrm{a}_{2} \mu\left(\mathrm{F}_{E N}\right)+\mathrm{a}_{3} \mu\left(\mathrm{F}_{W I N D}\right) \\
\text { s.t. } a_{1}+a_{2}+a_{3}=1 \\
F+\mu(\mathrm{F}) \alpha \mathrm{F}^{\min } \leq \mathrm{F}^{\min }+\alpha \mathrm{F}^{\min } \\
G(\mathrm{x}) \leq 0 \\
H(\mathrm{x})=0
\end{array}\right.
$$

The Pareto frontier can be solved efficiently by traversing the weights $0 \leq \alpha_{1} \leq 1,0 \leq \alpha_{2} \leq 1-\alpha_{1}$, $\alpha_{3}=1-\alpha_{1}-\alpha_{2}$. This method not only has the advantage of fuzzy planning, but also takes into account the tolerance limit of scheduling decision makers for different objective functions, and the normalization process in the satisfaction function makes the distribution of non-inferior solutions obtained from the traversal weights more uniform. Meanwhile, the scalarization process speeds up the solution speed and guarantees the accuracy of the non-inferior solutions.

\subsection{Pareto non-inferiority screening based on evidential reasoning}

Among all the Pareto non-inferior solutions, a small set of Pareto candidate solutions can be selected by calculating the distance from the ideal point $X=\left\{x_{1}, x_{2}, \ldots, x_{n}\right\}$, and $\mathrm{N}$ is the total number of candidate solutions. The most reasonable scheduling solution is obtained by multi-attribute decision based on evidence, which is [14]: firstly, we analyze the candidate solutions by multi-objective attributes, and then we define the evaluation indexes of the candidate solutions as different objective functions, and the evaluation 
grades as their relative advantages and disadvantages. According to the specific value of the evaluation index of the candidate solution, the confidence level of each evaluation index will be given, so the confidence level of each evaluation index will form the set of evidence for the utility evaluation, which is as follows:

$$
S\left(\mathrm{x}_{n}, \mathrm{y}_{m}\right)=\left\{\left(z_{l}, \xi_{n, m, l}\right)\right\}, n \in N, m \in M, l \in L+1
$$

$\mathrm{M}$ is the total number of evaluation indicators, the number of objective functions in the text; $\mathrm{L}$ is the total number of evaluation levels; $Z_{l}$ is the evaluation rating, which in the text is specifically \{poor, poor, fair, good, excellent $; y_{m}$ is specific evaluation indicator; $\xi_{n, m, l}$ is the confidence level, with specific values from 0 to 1 . $\sum_{l=L} \xi_{n, m, l}$ can reflect the level of awareness of an evaluation indicator by the scheduling decision maker, with 0 indicating that it cannot be evaluated, 1 indicating that it can be fully evaluated and 0 to 1 indicating an incomplete evaluation. To reflect the incompleteness of the decision maker's information, the value in the text is 0.9 , which means that the decision maker has a 0.1 uncertainty confidence level for each evaluation indicator. The above multidimensional evidence set was then fused and simplified.

$$
S\left(\mathrm{x}_{n}\right)=\left\{\left(z_{l}, \xi_{n, l}\right)\right\}, n \in N, l \in L+1
$$

A utility function is used to map the evidence vector to a specific utility assessment metric, with higher utility values indicating better multi-objective comprehensive performance, and the candidate solution with the highest comprehensive utility is selected as the optimal scheduling decision.

First define $\mu\left(Z_{1}\right)$ as the specific utility of the $l$ evaluation level, the value of which is obtained by the probability distribution method [15]. Considering the incomplete assessment of the scheduling decision maker, the specific utility function is given by :

$$
\begin{aligned}
& u_{\max }\left(\mathrm{x}_{n}\right)=\sum_{l=1}^{L-1} \xi_{n, l} u\left(\mathrm{z}_{1}\right)+\left(\xi_{n, \mathrm{~L}}+\xi_{n, \mathrm{~L}+1}\right) \mathrm{u}\left(\mathrm{z}_{L}\right) \\
& u_{\min }\left(\mathrm{x}_{n}\right)=\left(\xi_{n, 1}+\xi_{n, \mathrm{~L}+1}\right) \mathrm{u}\left(\mathrm{z}_{1}\right)+\sum_{l=2}^{L} \xi_{n, l} u\left(\mathrm{z}_{l}\right) \\
& u\left(\mathrm{x}_{n}\right)=\frac{u_{\max }\left(\mathrm{x}_{n}\right)+\mathrm{u}_{\min }\left(\mathrm{x}_{n}\right)}{2}
\end{aligned}
$$

\section{Analysis of the algorithm}

\section{(1) Background of the case}

To verify the validity of the model proposed in this paper. A park in northern China is selected for simulation. The park is rich in renewable energy and has a diverse demand side. We set up two scenarios in the arithmetic analysis, one considering the operation optimization model proposed in the paper and the other not considering the operation optimization of the normal operation of the park. The integrated energy structure of the park is coupled with electricity and heat; the electric system includes a gas turbine and a large power grid; the heat system includes a gas turbine, a gas boiler, a vacuum boiler and a heat storage tank. The predicted electric and thermal load curves for a typical day in the park are shown in Fig.2.

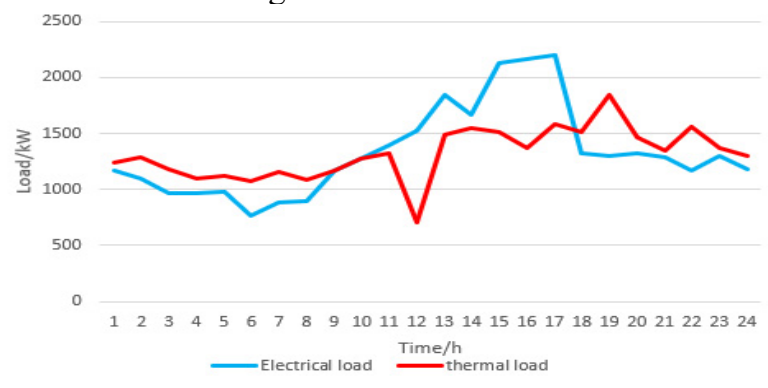

Fig.2 Predicted electric and thermal load curves for a typical day in the park.

(2) Analysis of results

1) RIES electrical system supply-demand balance diagram considering IDSR.

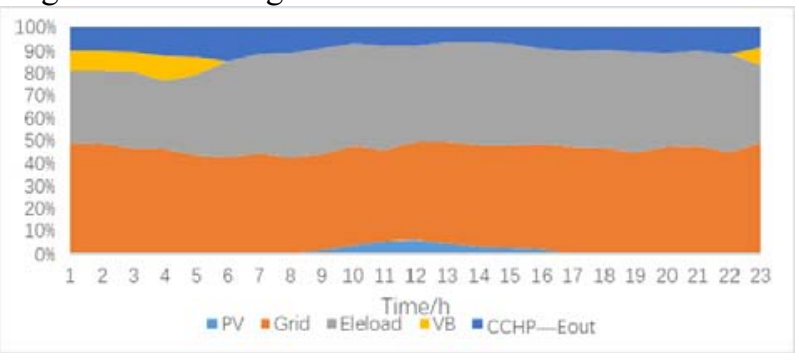

Fig.3 RIES electrical system supply and demand balance diagram considering the IDSR

2) Supply and demand diagram for the RIES heat system considering IDSR.

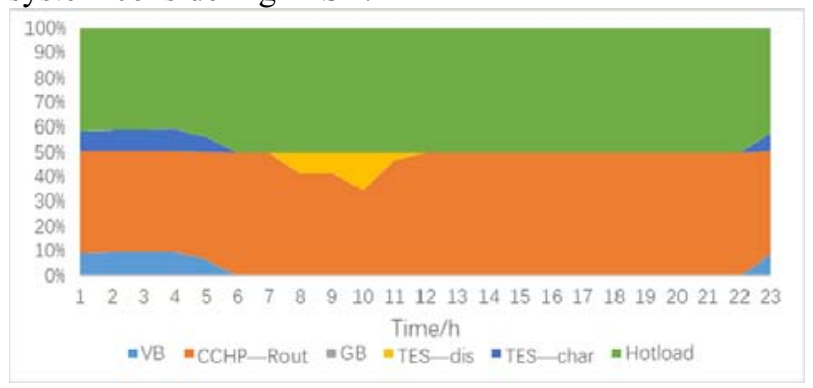

Fig. 4 Supply and demand diagram of the RIES thermal system considering IDSR

Table 1 Optimization Results of Multi-Objective Operation for Two Scenarios

\begin{tabular}{|c|c|c|c|}
\hline \multirow{2}{*}{ scene } & \multicolumn{3}{|c|}{ Optimized results for multi-objective operations } \\
\cline { 2 - 4 } & Economy/¥ & efficiency & Environment/¥ \\
\hline 1 & 1728695.36 & 1.89 & 1764.53 \\
\hline 2 & 1633459.12 & 1.32 & 2341.66 \\
\hline
\end{tabular}

The scheduling results for the two scenarios are compared, as shown in Table 1. Table 1 shows that the integrated demand-side response has a significant effect on the economic, energy efficiency and environmental aspects of RIES. The introduction of integrated demand- 
side response in RIES resulted in a 5.56\% reduction in total operating costs, a $30.15 \%$ increase in energy efficiency, and a $24.62 \%$ reduction in environmental protection costs. The benefits of introducing demand response to the overall costs are relatively modest, as the implementation of integrated demand response requires subsidies to users, which places a burden on the total costs of RIES.

\section{Conclusion}

The proposed multi-objective optimization scheduling model and corresponding solution method are proposed under the framework of RIES, taking into account the scheduling needs of RIES in terms of economy, environment and wind power consumption, and analyzing the influence of the synergistic effect of multiple energy devices within RIES and the integrated demand response on multi-objective optimization. Finally, the proposed method is verified to be effective in making trade-offs among multiple dispatching objectives in RIES, with fast solution speed and optimal global results. At the same time, the integrated demand response has a positive effect on the performance of the system. The next step will be to address how to quickly solve the non-convex model for multi-objective optimization.

\section{Acknowledgment}

This paper is supported by "Science and Technology Foundation of State Grid Corporation of China (SGHEJY00NYJS2000055)".

\section{References}

1. LU Zongxia, LI Haibo, QIAO Ying. Flexibility evaluation and supply/demand balance principles of power system with high penetration renewable electricity[J]. Proceedings of the CSEE, 201737 (1): 9-19.

2. LI Haibo, LU Zongxiang, QIAO Ying, et al. Assessment on operational flexibility of power grid with grid-connected large-scale wind farms[J]. Power System Technology, 2015, 39 (6): 1672-1678.

3. CESE AEAM, CAPUDER T, MANCARELLA P. Flexible distributed multi energy generation system expansion planning under uncertainty[J]. IEEE Transactions on Smart Grid, 2016, 7 (1): 348-357 .

4. SHEIKHI A, RAYATI M, BAHRAMIS, et al . Integrated demand side management game in smart energy hubs [J]. IEEE Transactions on Smart Grid, 2015, 6(2): 675-683.

5. WEI Zhenbo, HUANG Yuhan, GAO Hongjun, et al . Joint economic scheduling of power-to-gas and thermoelectric decoupling CHP in regional energy Internet[J]. Power System Technology, 2018, 42(11): 3512-3520.
6. DONG Shuai, WANG Chengfu, LIANG Jun, et al . Multi-objective optimal day-ahead dispatch of integrated energy system considering power-to-gas operation cost $[\mathrm{J}]$. Automation of Electric Power Systems, 2018, 42(11): 8-15, 121.

7. DONG Fugui, ZHANG Ye, SHANG Meimei. Multi-criteria comprehensive evaluation of distributed energy system [J]. Proceedings of the CSEE, 2016, 36(12): 3214-3223.

8. HU Xiao, SHANG Ce, CHEN Dongwen, et al. Multi-objective planning method for regional integrated energy systems considering energy quality[J]. Automation of Electric Power Systems, 2019, 43(19): 22-38, 139.

9. ZHENG Zhanghua, AI Qian GU Chenghong, et al. Multi-objective allocation of distributed generation considering environmental factor[J]. Proceedings of the CSEE, 2009, 29(13): 23-28.

10. WEI Zhenbo, HUANG Yuhan. Decentralized coordinated dispatch for multiple integrated electricity-gas energy systems considering demand side management $[\mathrm{J}]$. Electric Power Automation Equipment, 2019, 39(8): 277-285.

11. CLEGG S, MANCARELLA P. Integrated modeling and assessment of the operational impact of powerto-gas $(\mathrm{P} 2 \mathrm{G})$ on electrical and gas transmission networks[J]. IEEE Transactions on Sustainable Energy, 2015, 6(4): 1234-1244. 